\title{
Growth Failure and Obesity-The Twin Epidemic
}

\author{
Ram K. Menon ${ }^{1}$ Anurag Bajpai ${ }^{2}$
}

Published online: 21 October 2021

(c) Dr. K C Chaudhuri Foundation 2021

Disorders of growth and obesity are the most common endocrine disorders presenting to the pediatricians. Their systematic management requires timely identification, thorough assessment, and rational treatment. The growth and obesity symposium in this issue of the Journal covers key aspects of evaluation and management of growth and obesity-related disorders.

Rational evaluation of short stature aims at minimizing costly laboratory and investigational workup while maximizing detection of pathological causes. Patel et al. propose a practical approach based on a combination of auxological parameters, clinical pointers, and bone age that allows costeffective evaluation of children and adolescents with growth failure [1]. Growth hormone (GH) has been the mainstay of managing growth hormone deficiency (GHD), with increasing use in non-GHD conditions. Vyas et al. present the state of the art of GH treatment in children with GHD and beyond [2]. The therapeutic options for short children have expanded beyond GH with the advent of GnRH analogs, aromatase inhibitors, and, recently, CNP receptor agonists. Saroufim et al. provide much-needed clarity on the current use of these agents, providing a glimpse of futuristic growth therapeutics [3].

Obesity has emerged as a major public health problem and has replaced short stature as the most common presentation to a Pediatric Endocrinologist. Given that the overwhelming majority of children and adolescents presenting to the pediatricians represent exogenous and lifestyle-related obesity, the key is the targeted endocrine workup in at-risk individuals. Khadilkar et al. provide insights into the evaluation of childhood obesity with particular emphasis on clinical evaluation [4]. The management of childhood obesity is challenging, as it primarily involves lifestyle modifications. Pharmacological

Anurag Bajpai

dr_anuragbajpai@yahoo.com

1 Division of Endocrinology, Department of Pediatrics, CS Mott Children Hospital University of Michigan, Ann Arbor, MI 48109, USA

2 Department of Pediatric \& Adolescent Endocrinology, Regency Center for Diabetes, Endocrinology \& Research, Kanpur, Uttar Pradesh 208001, India and surgical measures should play secondary roles but become indicated in many patients. Mittal et al. have presented a comprehensive overview of preventive and therapeutic strategies for childhood obesity and its complications [5].

Small for gestational age represent an endocrine conundrum with myriad effects ranging from growth failure to precocious pubarche and metabolic syndrome. The effects are especially pertinent to resource-poor countries with a high prevalence of the condition highlighting the need for balance between growth promotion and prevention of metabolic complications. Cutfield et al. summarize the current evidence about the endocrine effects of small size at birth along with their evidence-based management [6].

The symposium presents current evidence-based evaluation and management of growth failure and obesity in children and adolescents.

\section{Declarations}

Conflict of Interest None.

\section{References}

1. Patel R, Bajpai A. Evaluation of short stature in children and adolescents. Indian J Pediatr. 2021. https://doi.org/10.1007/ s12098-021-03880-9.

2. Vyas V, Menon RK. Management of short stature: use of growth hormone in GH-deficient and non-GH-deficient conditions. Indian J Pediatr. 2021. https://doi.org/10.1007/s12098-021-03892-5.

3. Saroufim R, Eugster EA. Non-GH agents and novel therapeutics in the management of short stature. Indian J Pediatr. 2021. https:// doi.org/10.1007/s12098-021-03824-3.

4. Khadilkar V, Shah N. Evaluation of children and adolescents with obesity. Indian J Pediatr. 2021. https://doi.org/10.1007/ s12098-021-03893-4.

5. Mittal M, Jain V. Management of obesity and its complications in children and adolescents. Indian J Pediatr. 2021:1-13. https://doi. org/10.1007/s12098-021-03913-3.

6. Cutfield W, Ayyavoo A. The auxological and metabolic consequences for children born small for gestational age. Indian J Pediatr. 2021. https://doi.org/10.1007/s12098-021-03897-0.

Publisher's Note Springer Nature remains neutral with regard to jurisdictional claims in published maps and institutional affiliations. 